\title{
Prevalence of aortic arch anatomical branching variants in Kenyan population as shown on contrast enhanced chest multidetector computed tomography
}

\author{
Daniel M. Amakabane, Gladys N. Mwango \\ Department of Diagnostic Imaging and Radiation Medicine, University of Nairobi, Nairobi, Kenya \\ Correspondence: Dr Daniel M. Amakabane (amakabaned@yahoo.com)
}

C 2018 D.M. Amakabane \& G.N. Mwango. This open access article is licensed under a Creative Commons Attribution 4.0 International License (http://creativecommons.org/licenses/by/4.0/) which permits unrestricted use, distribution, and reproduction in any medium, provided you give appropriate credit to the original author(s) and the source, provide a link to the Creative Commons license, and indicate if changes were made.

East Cent Afr J Surg. 2018 Aug;23(2):71-75 https://dx.doi.org/10.4314/ecajs.v23i2.4

\begin{abstract}
Background

The objective of the study was to determine the prevalence of aortic arch(AA) anatomical branching variants present in Kenyan population as shown on a chest contrast-enhanced multidetector computed tomography (MDCT).

\section{Methods}

The study design was a prospective cross-sectional study carried out in 6 months (between May and November 2016) at the Kenyatta National Hospital(KNH) department of Radiology. Participants were recruited amongst patients referred for a chest contrast enhanced MDCT. Acquired data was subjected to volume rendering technique (VRT) and multiplanar reconstruction (MPR) software to define the anatomy of the AA.

The type of AA branching classification used was one derived by Natsis et al.

Data analysis involved calculating frequency distributions of AA variations using Statistical Package for Social Sciences version 21 software.

\section{Results}

One hundred and eighty-five subjects (86 males and 99 females) were recruited. Ninety-five participants had classical AA Natsis type I. Ninety participants had non-classical variant AA of which 83 participants had Natsis type II while 2 patients had Natsis type III. The remaining 3 had a variant AA not found in the Natsis study.
\end{abstract}

\section{Conclusions}

Variations in the branching pattern of AA are very common in Kenya with the most common being Natsis type II.

Keywords: aortic arch, anatomical variants, branching variation, chest multidetector computed tomography

\section{Introduction}

The aortic arch (AA) develops in a complex way and this intricacy predisposes it to various configurations. However, the normal or conventional or classical AA is left-sided and the commonest branching pattern of the AA consisting of three vessels; first the brachiocephalic trunk (BCT), then the left common carotid artery (LCCA), and finally the left subclavian artery (LSCA) from right to left. The BCT branches into the right subclavian artery (RSCA) and right common carotid (RCCA). This branching pattern occurs in $64.9-$ $94.3 \%$ of the populations around the world as reported by various authors in literature. ${ }^{1}$
Variations in the branching pattern of the AA include differences in the origins of different branches and the number of branches. This has clinical and anatomical significance to diagnostic and interventional radiologists, cardiothoracic, vascular, head and neck surgeons. The non-classical AA branching variants have been found to be associated with a higher occurrence of congenital cardiovascular malformations amongst these patients. ${ }^{2}$

Locally the anatomy of the aortic arch has only been studied by cadaver dissection. ${ }^{3}$ In other regions of the world other methods including conventional angiography, digitally subtracted angiography (DSA), computed tomography angiography (CTA) and multidetector computed tomography (MDCT) have been used in addition to cadaver dissection 
to study the anatomy of the AA. In this study the anatomy of the AA in a Kenyan population using the contrast-enhanced MDCT of the chest was used. From the literature review no such study using contrast enhanced MDCT has been reported in Kenya and Africa. Hence this will form baseline radiological data for comparison and for similar future studies in this topic.

The classification described by Natsis et al (2009) was used in this study because it is radiological based and is the most recent in literature. ${ }^{4,5}$ In this classification the anatomy of the AA vessels as seen on digital subtraction angiography was categorized into 8 types.

Knowledge of AA branching is clinically important because some of these variants e.g. the Natsis type II AA are known to be associated with a higher incidence of congenital cardiovascular diseases. ${ }^{6}$ It is also associated with risk of retrograde aortic dissection during or after thoracic endovascular aortic repair (TEVAR). The occurrence rates ranges from $1 \%$ to $3 \%$.

Although AA anatomic branching variants are largely asymptomatic some, e.g., aberrant right subclavian artery (ARSCA) may cause compression to the trachea and esophagus and lead to respiratory disturbances (dyspnea) and dysphagia (dysphagia lusoria). ${ }^{3,8}$ ARSCA can be fatal in symptomatic infants and their timely diagnosis could be lifesaving. If the ARSCA courses anterior to the trachea it may cause great danger in a patient who requires an emergency tracheostomy. Hence prior knowledge about or anticipation of these variant is essential to avoid injury to the vessel.

Other variants can lead to intermittent claudication, misinterpretation of radiological examination and complications during neck and thoracic surgery.

As shown by Faggioli et al in their study, non-classical AA anatomical variants are associated with a higher risk of neurological complications in carotid stent procedures and technical failure was more in the same group of patients. ${ }^{9}$

\section{Methods}

The aim of this study was to investigate the prevalence of AA anatomical branching variations in patients who undergo contrast enhanced chest MDCT scans for various reasons at $\mathrm{KNH}$ department of Radiology. This was achieved through the following specific objectives:

1. To determine the prevalence of AA branching variants as depicted by chest enhanced MDCT scans.

2. To determine gender distribution of these variants in Kenyan population

3. To describe the patterns of aortic arch branching variations depicted by chest enhanced MDCT scans.

Chest CT scan images were acquired by the "Siemens SOMATOM Definition AS+ 128 slice Multidetector Row CT scanner (Siemens AG, Munich, Germany)".

For adults the following protocol was used $; 120 \mathrm{kV}$, up to 200 effective (mAS),128x0.6mm collimation, pitch $0.9,3$ $\mathrm{mm}$ section slices, reconstruction interval $=0.8 \mathrm{~mm}$, tube rotation period $=0.5$ seconds. The field of view was adjusted to the size of the patient. Topogram length of $512 \mathrm{~mm}$ was used. This was from the apices of the lungs extending to the lowest hemidiaphragm. Scans in full inspiration were obtained. Intravenous contrast consisting of a volume of $60-70 \mathrm{ml}$ of iodine based, non-ionic contrast material $(300 \mathrm{mg} \mathrm{I} / \mathrm{ml})$ injected through an antecubital vein by an injector pump at a rate of $2.5 \mathrm{ml} / \mathrm{s}$. 5 seconds delay time was allowed. Scan time was 5.91 seconds

For pediatric patients a low dose radiation CT protocol was used which included $110 \mathrm{kV}, 35 \mathrm{mAS}, 128 \times 0.6 \mathrm{~mm}$ collimation, pitch 1.4, slice thickness of $6 \mathrm{~mm}$, delay time of 2 seconds, a topogram length of $256 \mathrm{~mm}$, and the contrast medium calculated at a rate of $1 \mathrm{ml} / \mathrm{kg}$ body weight of the patient. The contrast medium was injected at a rate of $1 \mathrm{ml} / \mathrm{s}$. Scan time was 4.1 seconds.

Raw and reformatted data MDCT scans of every patient were examined and subjected to the MPR and VRT software by a consultant radiologist and the principal investigator to confirm the AA branching pattern.

Biodata was collected by the principal researcher and the trained data clerks recruited among the radiographers stationed at the Siemens CT room where chest MDCT examinations are performed.

Patients who gave informed consent were recruited into the study according to the inclusion criteria. There after the acquired images were reviewed, processed, reformatted and analyzed by principal researcher together with a consultant radiologist. The raw data for patients with non-classical variant AA were stored on DVD discs.

Data analysis involved calculating the number and frequencies of different aortic arch configurations related to the whole group of participants as well as among the participants with different anatomical aortic arch variations as well as in relation to gender.

The Statistical Package for Social Sciences (SPSS) version 21 software was used for data analysis.

Study period was 6 months extending between the months of May and November 2016.

The sample size was determined by Cochran formula $(1963)^{10}$ that gave a minimum of 181 patients to be included in the study. However, 185 patients were recruited into the study.

Before embarking on the study and collection of data approval was obtained from Kenyatta National Hospital-University of Nairobi Ethics and Research Committee (KNH-UoN ERC). This was granted on 18th May 2016. The reference number for this approval was KNH-ERC/A/167.

Informed consent to use images for this study was obtained from the participants after a full explanation by the principal investigator and the research assistants. All the studies were justified and the set study protocols as per requested investigation were followed strictly to ensure only minimum optimum radiation dose was given to the subjects to ensure radiation safety. Confidentiality was strictly observed by ensuring that patient's names were not quoted anywhere in the write up or in any discussion of the images and study findings. 


\begin{tabular}{|c|c|c|}
\hline Natsis type & $\begin{array}{c}\text { No of } \\
\text { patients }\end{array}$ & $\begin{array}{l}\text { Frequency } \\
(\%)\end{array}$ \\
\hline I & 95 & 51.3 \\
\hline II & 83 & 44.9 \\
\hline III & 4 & 2.2 \\
\hline Non Natsis types & 3 & 1.6 \\
\hline Total & 185 & 100 \\
\hline \multicolumn{3}{|c|}{$\begin{array}{l}\text { Table 2. NATSI classification of non-classical AA branch- } \\
\text { ing variants visualized using MDCT in study participants }\end{array}$} \\
\hline Natsis type & $\begin{array}{l}\text { No of } \\
\text { patients }\end{array}$ & $\begin{array}{c}\text { Frequency } \\
\text { (\%) }\end{array}$ \\
\hline II & 83 & 44.9 \\
\hline III & 4 & 2.2 \\
\hline Non Natsis types & 3 & 1.6 \\
\hline Total & 90 & 48.7 \\
\hline
\end{tabular}

\section{Results}

The study was performed among 185 participants who included pediatric and adult patients undergoing contrast enhanced chest MDCT at KNH department of Radiology. Of these participants 86 (46.5\%) were males and 99 (53.5\%) were females giving a male-to-female ratio of approximately $1: 1$.

The mean age of the participants was 50.7 years $(\mathrm{SD} \pm$ 18.4). (range 3 years to 93 years). The mean age of female participants was 49.2 years (SD \pm 19.4$)$ and the mean age of male participants was 52 years $(\mathrm{SD} \pm 17.9)$.

\section{Prevalence of AA branching variants among study participants}

The majority of the participants 51.3\% (95/185) had the classical vascular branching pattern Natsis type I of three branches consisting of BCT, LCCA and LSCA. This is as shown in table 1 below.

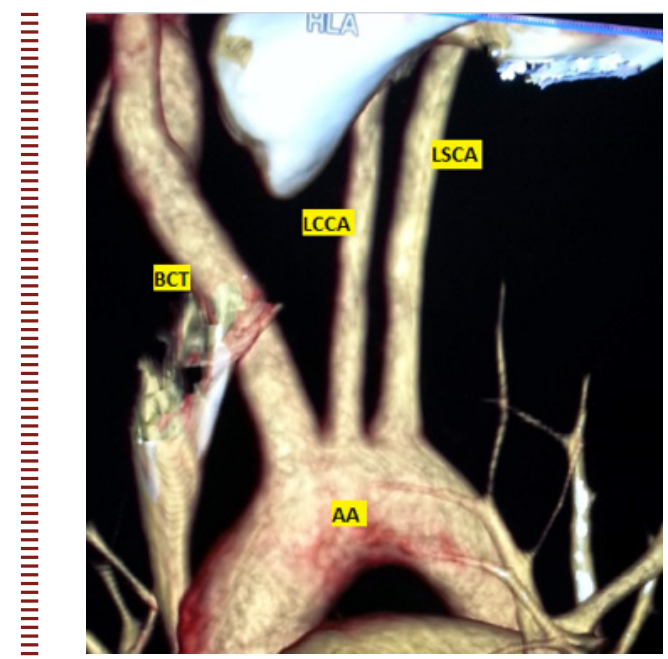

Figure 1. NATSIS TYPE I. Anterior coronal 3D volume-rendered MDCT image.The classical AA vascular branching pattern with 3 branches i.e. BCT, LCCA and LSCA

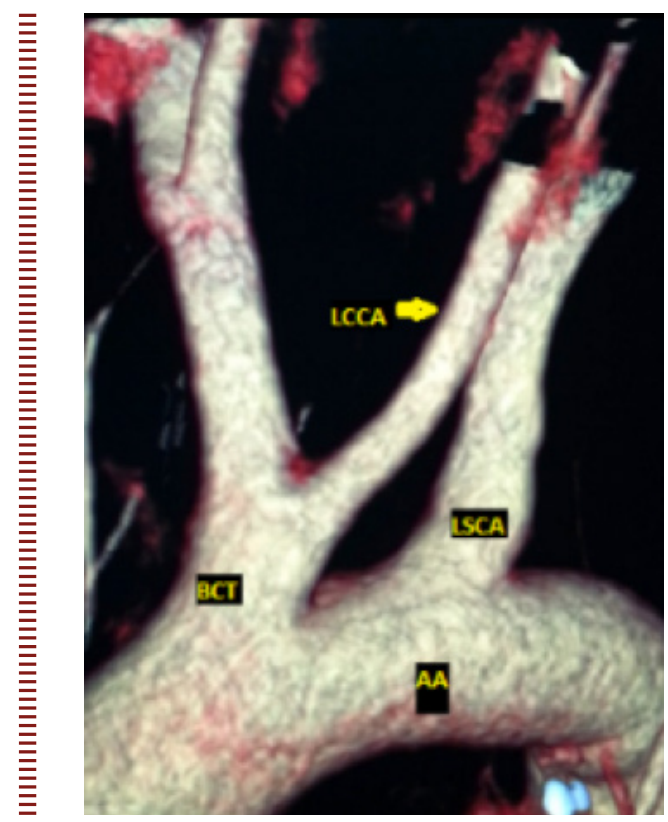

Figure 2. NATSIS TYPE II. Anterior coronal 3D volumerendered MDCT image. This pattern consists of two branches $B C T$ and LCSA. The LCCA originates from BCT instead of directly from the AA.

Table 2. NATSI classification of non-classical AA branching variants visualized using MDCT in study participants

\begin{tabular}{c|c|c|c|c}
\hline & \multicolumn{2}{|c|}{ Males } & \multicolumn{2}{c}{ Females } \\
\hline Types of variants & Participants(n) & Frequency (\%) & Participants(n) & Frequency (\%) \\
\hline \hline I & 46 & 53.3 & 49 & 49.5 \\
II & 36 & 41.9 & 47 & 47.5 \\
III & 3 & 3.5 & 1 & 1 \\
Non Natsis type & 1 & 1.2 & 2 & 2 \\
\hline Total & 86 & 100 & 99 & 100 \\
\hline
\end{tabular}




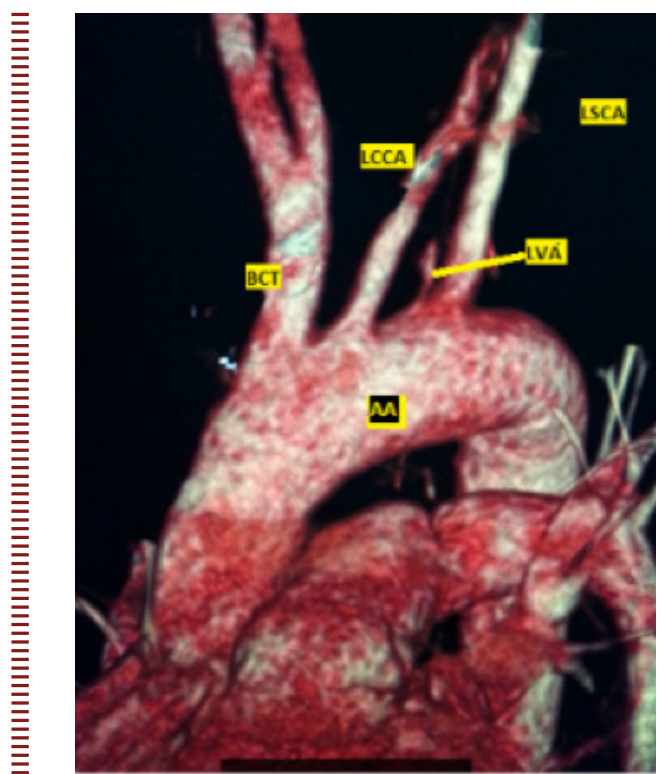

Figure 3. TYPE III. Anterior coronal 3D volume-rendered MDCT image. The AA has four branches, BCT, LCCA, LVA, LSCA where LVA originates directly from the AA between the LCCA and LSCA.

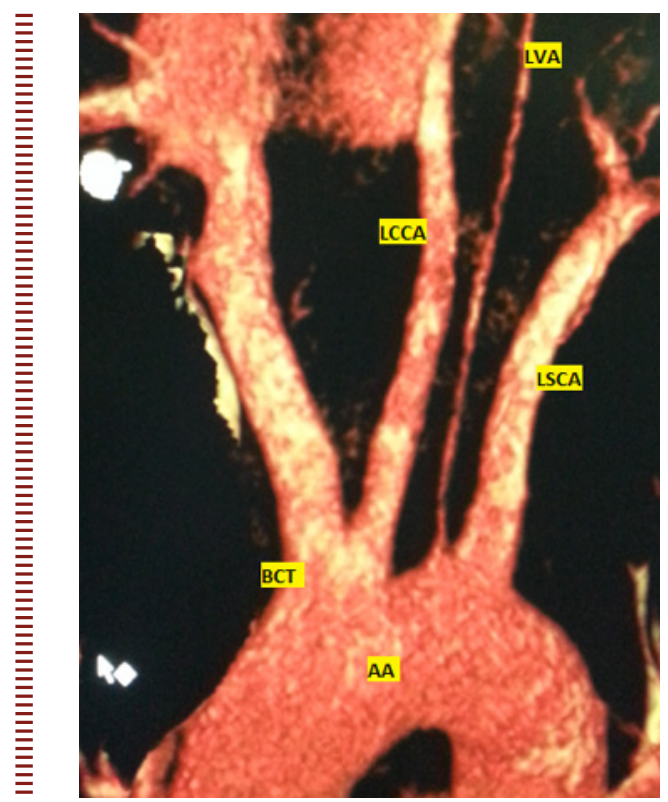

Figure 4. NON NATSIS TYPE. Anterior coronal 3D volumerendered MDCT image. The AA has three branches BCT, LVA and LSCA. The BCT gives rise to LCCA while the LVA originates directly from the AA between the BCT and LSCA.

The overall prevalence of non-classical vascular pattern of AA branching was $48.7 \%(90 / 185)$. The prevalence of Natsis type II was highest at $44.9 \%(83 / 185)$. This is as shown in table 2 below.

\section{Gender distribution of aa branching variants}

Classical Natsis type I variant was found in 46 male participants (53.3\%) and 49 of female participants (49.5\%). Non-classical variants were found in 40 males (46.7\%) and 50 female participants $(50.3 \%)$. This is as shown in table 3 below.
Overall, there was no statistically significant association between AA branching variants and gender $(\chi 2=1.98, \mathrm{p}=$ 0.576)

\section{AA branching patterns as depicted by MDCT}

The AA branching patterns demonstrated in the study are represented below by volume-rendered MDCT image of 4 patients (participants).

\section{Discussion}

In this study the classical or conventional or normal three branch pattern Natsis type I was observed in $51.3 \%$ of the cases. This falls below the incidence found in most studies world wide of between 64.9- $94.3 \%$ according to literature. ${ }^{1}$ However, this falls within 2 studies cited by Nurefsan in his literature review which reported that the incidence of Natsis type I varied between 49.7 and $51.7 \%$ in African Americans. ${ }^{11}$ The remaining $48.7 \%$ of the participants had non-classical variants. This is a higher incidence when compared to that found in most literature of 5.7-35.1\% but still coincides with the above studies reported by Nizankowski C and Williams $\mathrm{GD}^{7}$ and the other reports by McDonald and Anson that deviation from classical AA branching is more common in African populations. ${ }^{1}$

The most common non-classical variant of the AA was Natsis type II with two branches ([BCT sharing one AA origin with LCCA] and LSCA) is found with an incidence of $44.9 \%$. This is within the range reported in studies which report varying prevalence rates ranging between 0.9 and $45.6 \%$. The highest reported rate was by a study on American Africans and American Caucasians by Williams and Henry of $45.6 \%{ }^{11}$ Two Turkish studies reported prevalence of $2.6 \%$ and $7.2 \%{ }^{1,11}$ with much larger sample sizes of 1170 and 881 patients respectively. This difference may also be attributed to genetic differences in study populations.

Natsis type III was observed in $2.2 \%$ of my study population which compares well to the prevalence reported in literature of between $2.4-8.0 \% .^{1,2,4,6,11}$ However, the frequency is higher than that reported by Ogengo et al. ${ }^{3}$ in the earlier local cadaver dissection study (0.9\%) from a similar population. The reasons for this difference remains unclear. However, in both Kenyan studies, there was agreement in the absence of Natsis IV -VII. These later types have been reported in European subcontinent. ,, 69

The non-Natsis variants were seen in $1.6 \%$ of study participants. Comparable prevalence was reported in the South African population by Makhanya et al of $1.7 \%$ The racial structure is not mentioned in this study. ${ }^{12}$ This prevalence also compares closely to that reported in a Central Indian population by Budhiraja et al. ${ }^{13}$ Similar variants in Turkish population have been reported with a frequency of $1.1 \%^{6}$ and in British population with a frequency of $0.2 \% .{ }^{4}$ It is postulated that the non-Natsis branching pattern and the Natsis type II have a higher incidence in the African study populations. ${ }^{12}$ 
This study did not demonstrate any significant gender difference amongst the participants a finding that is found in most study populations around the world.

\section{Study limitations}

Lack of a Picture Archiving and Communication System (PACS) at the KNH department of Radiology for storing patient information including images prevented retrospective data collection. Storage of patients' data in a PACS would have yielded larger samples of participants as seen with similar studies elsewhere in the world.

\section{Conclusions}

Variations in the branching pattern of AA are very common in Kenya with the most common being Natsis type II. This prevalence is higher as compared to the local study by Ogeng'o et al carried by cadaver dissection. This difference could be due to sampling technique.

Knowledge of these variations is important for cardiothoracic, head and neck surgeons and interventional radiologists to be aware so as ensure safer and more accurate endovascular and surgical planning

\section{Recommendations}

A prospective study should be undertaken to investigate the incidence of congenital cardiac anomalies in the Kenyan population considering the relative higher prevalence of AA variants at $48.7 \%$ depicted by this study.

A prospective study should be undertaken to investigate the pathologies and/or perfusion abnormalities if any that could be associated with these variants in the Kenyan population.

Installation of a picture archiving and communications system (PACS) for storage and archiving of patient data and images should be installed at Kenyatta National Hospital as a matter of priority as it would facilitate patient follow-up and research.

\section{Acknowledgements}

We thank Dr Beatrice Mugi (consultant radiologist $\mathrm{KNH}$ Radiology Department) whom we studied all the images obtained to confirm the AA configuration and Mr. Philip Ayieko who offered statistical assistance in calculation and determination of the sample size and in the analysis of the data.

\section{Competing interests}

Both authors declare that they have no competing interests related to this work.

\section{References}

1. Lale P, Toprak U, Yagız G, Kaya T, Uyanık SA. Variations in the branching pattern of the aortic arch detected with computerized tomography angiography. Adv Rad. 2014;969728. doi: 0.1155/2014/969728.

2. Vučurević G, Marinković $S$, Puškaš L, Kovačević I, Tanasković S, Radak D, et al. Anatomy and radiology of the variations of aortic arch branches in 1,266 patients. Folia Morphol (Warsz). 2013;72(2):113-22. doi: 10.5603/FM.2013.0019.

3. Ogeng'o JA, Olabu BO, Gatonga PM, Munguti JK. Branching pattern of aortic arch in a Kenyan population. J Morphological Sci. 2010;27(2):51-5.

4. Jakanani GC, Adair W. Frequency of variations in aortic arch anatomy depicted on multidetector CT. Clin Rad. 2010;65(6): 481-7. doi: 10.1016/j.crad.2010.02.003.

5. Natsis $\mathrm{KI}$, Tsitouridis IA, Didagelos MV, Fillipidis AA, Vlasis GK, Tsikaras PD. Anatomical variations in the branches of the human aortic arch in 633 angiographies: clinical significance and literature review. Surg Radiol Anat. 2009 Jun;31(5):319-23. doi: 10.1007/s00276008-0442-2. Epub 2008 Nov 26.
6. Ergun $O$, Tatar IG, Birgi E, Durmaz HA Akçalar S, Hekimoğlu B. Variations in the branching pattern of human aortic arch; angiographic study on 270 patients [Poster C-1403] [Internet]. Abstracts of the European Congress of Radiology 2014. Vienna: European Society of Radiology; c2014. Available from: http:// pdf.posterng.netkey.at/download/index php? module=get_pdf_by_id\&poster_ id $=122209$.

7. Tapia GP, Zhu X, Xu J, Liang P, Su G, Liu $\mathrm{H}$, et al. Incidence of branching patterns variations of the arch in aortic dissection in Chinese patients. Medicine (Baltimore). 2015 May;94(17):e795. doi: 10.1097/ MD.00000000000000795.

8. Bhattarai C, Poudel PP. Study on the variation of branching pattern of arch of aorta in Nepalese. Nepal Med Coll J. 2010 Jun;12(2):84-6

9. Faggioli GL, Ferri M, Freyrie A, Gargiulo M, Fratesi F, Rossi C, et al: Aortic arch anomalies are associated with increased risk of neurological events in carotid stent procedures. Eur J Vasc Endovasc Surg. 2007 Apr:33(4):436-41. Epub 2007 Jan 19.
10. Cochran WG. Sampling techniques. 2nd ed. New York: John Wiley \& Sons; 1963.

11. Boyacı N, Dokumacı DŞ, Karakaş E, Yıldız S, Cece $\mathrm{H}$, Kocarslan A, et al. Multidetector computed tomography evaluation of aortic arch and branching variants. Turk Gogus Kalp Dama. 2015;23(1):51-7. doi: 10.5606/tgkdc.dergisi.2015.10290.

12. Makhanya NZ, Mamogale RT, Khan N. Variants of the left aortic arch branches. S Afr J Rad. 2004;8(4):10-2.

13. Budhiraja V, Rastogi R, Jain V, Bankwar V, Raghuwanshi S. Anatomical variations in the branching pattern of human aortic arch: a cadaveric study from central India. Int Sch Res Notices. 2013;8289691. doi: 10.5402/2013/828969. 